\title{
Longitudinal association between leisure-time physical activity and vascular elasticity indices
}

\author{
Gábor Szaló ${ }^{1,2}$, Margareta Hellgren², Matthew Allison ${ }^{3}$, Lennart Råstam4, Ulf Lindblad² and Bledar Daka²*
}

\begin{abstract}
Aim: We investigated the association between levels of leisure-time physical activity and vascular stiffness in a longitudinal observational study from a representative Swedish population.

Method: A total of 2816 randomly selected individuals were examined at visit 1 (2002-2005, Men=1400). After a mean follow-up of $9.7 \pm 1.4$ years, a representative sample of 1327 of the original participants were re-examined at visit 2. After excluding subjects with hypertension at baseline, 761 participants were included in the longitudinal analyses. Leisure-time physical (LTPA) activity was self-reported and dichotomized as high or low (level 3,4 and level 1, 2, respectively). Large Arterial Elasticity Index (LAEI) and Small Arterial Elasticity Index (SAEI) were measured using the HDI/Pulse Wave ${ }^{\text {TM }}$ CR2000. Multivariable general linear models were used to investigate the differences in changes SAEl and LAEI based on LTPA levels.

Results: At visit 1, and after adjustment for possible confounders, participants in the high LTPA group had better small artery elasticity (SAEI) (SAEl in low-level LTPA: $7.89 \pm 0.11$, SAEl in high-level LTPA: $8.32 \pm 0.15, \triangle S A E I: 0.42$, Cl: $0.07-0.78 ; p=0.020$ ). SAEl decreased between the two assessments (Visit 1: SAEl $8.01 \pm 3.37 \mathrm{ml} / \mathrm{mmHg} ; \triangle$ SAEl: 1.4 , CI 1.2-1.6, $p<0.001$ ). Participants with a higher LTPA at visit 1 had significantly better SAEl at visit 2 ( $\triangle$ SAEl: $0.44, \mathrm{Cl}$ $0.03-0.85, p=0.037)$. No significant associations were observed between LAEI and LTPA after adjustments.
\end{abstract}

Conclusions: High LTPA predicted higher small arterial compliance at visit 2 suggesting that positive effects of LTPA on arterial elasticity persists over time.

Keywords: Vascular aging, Leisure-time physical activity, Vascular function, Small artery elasticity index, Arterial compliance

\section{Introduction}

One of the most important goals in primary care is to identify individuals with increased cardiovascular risk. High blood pressure is the most robust risk factor for cardiovascular disease $[1,2]$. Blood pressure is considered to contain a steady component, mean blood pressure, and

\footnotetext{
*Correspondence: bledar.daka@allmed.gu.se

2 Primary Health Care, School of Public Health and Community Medicine, Institute of Medicine, Sahlgrenska Academy, University of Gothenburg, Gothenburg, Sweden

Full list of author information is available at the end of the article
}

a pulsatile component, the pulse pressure. Hemodynamic research has shifted away from studying steady flow toward investigating pulsatile flow to include measurement of arterial elasticity [3]. Measures of arterial elasticity provide extra prognostic information beyond arterial blood pressure measurement [4] and can be used both for early detection of vascular disease and identification of increased risk for cardiovascular diseases (CVD) [5].

Early atherosclerosis is characterized by endothelial dysfunction followed by structural changes [5] including increased arterial stiffness, particularly in the smaller arteries [6]. In this regard, arterial elasticity largely 
depends on the ratio of elastin to collagen in arterial walls. The main changes are represented by degeneration of elastin material and an increase in collagenous material, often accompanied by calcium deposition in the ground substance and degenerated elastic fibres [7]. Vascular elasticity can be measured by diastolic pulse contour analysis of the arterial waveform which is a reproducible and reliable method $[8,9]$. Duprez et al. evaluated systolic and diastolic pulse wave analysis and found that both of these indices correlated with the Framingham Risk Score [3]. In the Multi-Ethnic Study (MESA) Small Artery Elasticity Index (SAEI) was significantly associated with coronary disease, stroke, and heart failure [4].

In cross-sectional studies, it has been observed that individuals with low levels of physical activity have increased vessel stiffness, compared to active individuals [10-12]. According to a systematic review, aerobic exercise significantly increases vascular elasticity and the effect was better at higher exercise intensity and in participants with greater arterial stiffness at baseline [13]. To our best knowledge, there are only a few observations on how physical activity affects changes in vascular elasticity $[11,14,15]$ and studies in representative populations and long-term follow-up are still missing. Thus, this study aimed to investigate the relationship between physical activity and vascular elasticity with a specific focus on whether low levels of physical activity are associated with the development of vascular stiffness in a longitudinal observational study from a representative Swedish population without hypertension.

\section{Method \\ Study population}

Details on Skaraborg population study have been described in detail previously [16]. In brief, it is a longitudinal observational study based on representative participants from Vara and Skövde municipalities in south-western Sweden. Between 2002 and 2005, a total of 2816 randomly selected individuals were examined $($ Men $=1400)$ for early signs off cardiovascular disease and risk factors for the development of CVD. After 10 years (9.7 years), and between 2012 and 2014, a representative sample of 1327 people were re-examined according to the same protocol as at visit 1 (Fig. 1). Two hundred eighty-eight participants do not have data on (Leisure Time Physical Activity) LTPA or arterial elasticity at visit 1 , the corresponding number is 425 at visit 2 . The number of participants with known hypertension was 104 at visit 1 , and 141 at visit 2, respectively. Hypertensive medication can interfere with the elasticity indices significantly thus we chose to exclude participants with known hypertension at any of the visits. As such, in the cross-sectional analysis, 935 participants were included, while 761 participants were included in the longitudinal analysis at both visit 1 and 2 (Fig. 1).

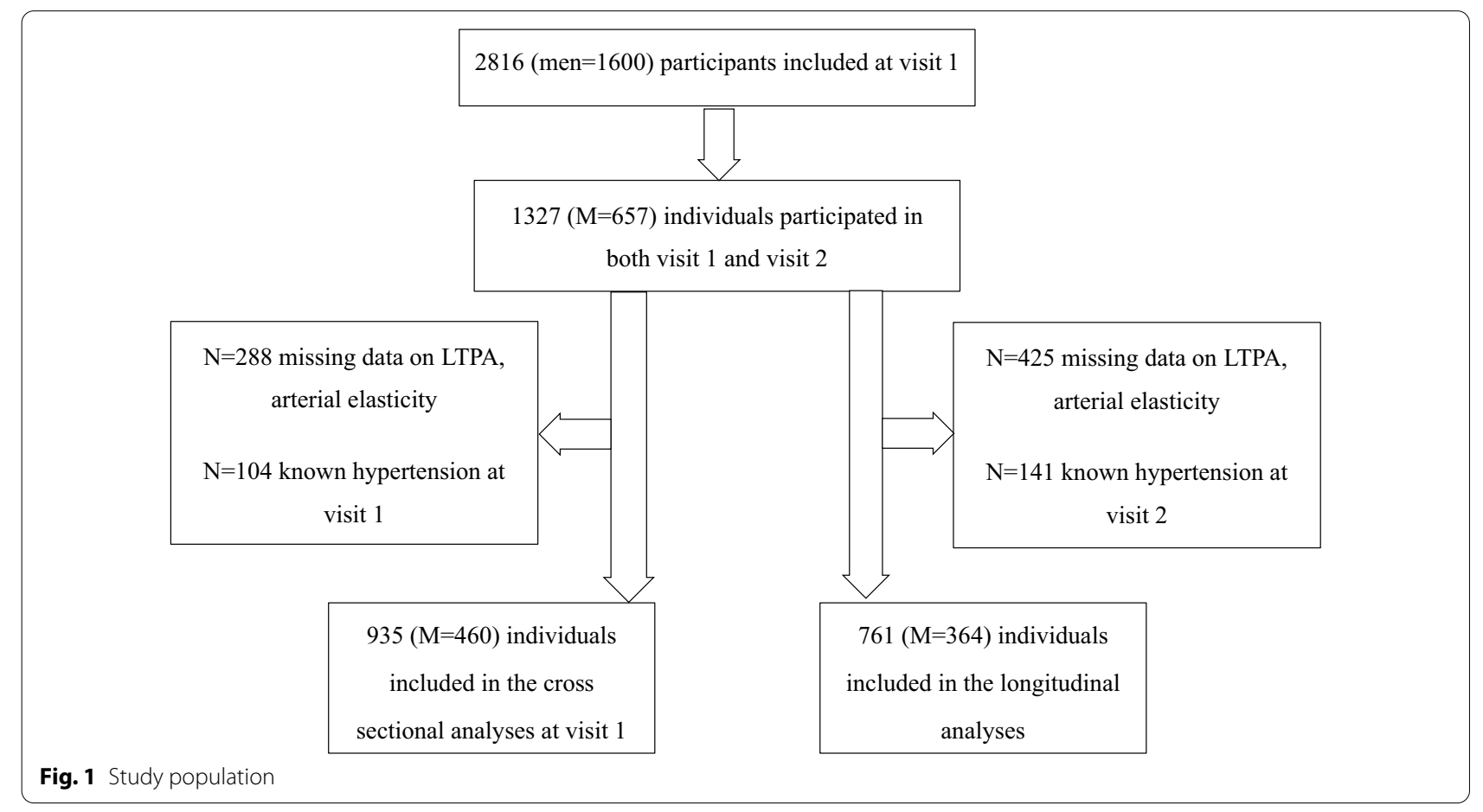




\section{Measurements}

Detailed information on the medical history including heart failure, atrial fibrillation, myocardial infarction, stroke, undergoing coronary heart surgery, as well as medication psychosocial and lifestyle was collected using validated questionnaires. All participants were asked alcohol [17] and smoking habits using validated questionnaires.

Body height and weight were measured in light clothes and no shoes to the nearest $1 \mathrm{~cm}$ and $0.1 \mathrm{~kg}$, while waistand hip circumferences were measured between the lowest rib margin and the iliac crest and at the largest circumference between waist and thighs, respectively. The blood pressure was measured to the closest $2 \mathrm{mmHg}$ in the right arm after a five minutes rest in a supine position $[16,18]$. Hypertension was defined based on Blood Pressure Management JNC-7 [19].

All participants underwent an oral glucose tolerance test (OGTT), whereby fasting and $2 \mathrm{~h}$ glucose values were obtained. Diabetes diagnosis was based on the 1999 WHO recommendation [20]. Fasting plasma insulin was analyzed using an enzyme-linked immunosorbent assay (ELISA) with $<0.3 \%$ cross-reactivity for proinsulin. Insulin resistance by HOMA-IR was calculated with the formula below [21]: fasting insulin (micro IU/L) $x$ fasting glucose $(\mathrm{nmol} / \mathrm{L}) / 22.5$. Assays for LDL cholesterol, triglyceride, C-reactive protein (CRP) and creatinine were conducted.

\section{Leisure-time physical activity}

The level of physical activity was estimated using a question about Leisure Time Physical Activity (LTPA) which has been validated in several previous studies [22-24]. All subjects were asked for their LTPA in the last 2 weeks. This scale has four levels based on four answer alternatives to the question "How much physical effort do you put yourself through in your leisure time?”.

1. Inactive, mostly physically inactive, and less strenuous LTPA (walking, cycling, gardening etc.) less than four hours a week $(\mathrm{h} / \mathrm{w})$;

2. Moderate, less strenuous LTPA $>4 \mathrm{~h} / \mathrm{w}$;

3. Strenuous, strenuous physical activity (jogging, swimming, tennis etc.) $>2 \mathrm{~h} / \mathrm{w}$ and

4. Highly strenuous, including those who participate in competitions on a weekly basis

\section{Pulse wave analyses}

Arterial compliance was measured based on diastolic pulse contour analysis of arterial waveform using the HDI/Pulse WaveTM CR2000 (Hypertension Diagnostics, Inc., Eagan, NY, USA). The tonometer was centered over the radial artery. The optimal radial artery waveform was obtained by non-invasive recording by applanation tonometry. This technique is based on a modified Windkessel model that gives an evaluation of the elasticity of the large conduit arteries (Large Arterial Elasticity Index, LAEI, C1) and the small microcirculatory arteries (Small Arterial Elasticity Index, SAEI, C2) [25, 26].

\section{Statistics}

Data were analyzed using SPSS 26. LTPA was grouped in 2 levels high and low. Subjects inactive and moderately active was classified as low LTPA and the others as high LTPA (high LTPA = level 3 and 4, low LTPA = level 1 and 2 ). General linear models were used to investigate the differences between these two groups in large and small elasticity indices at visit 1 and then changes over the two study visits. Adjustments for possible confounding were based on theoretical models, stepwise with each model building upon the previous one. In model, 1 we adjusted for age, sex, heart rate and systolic blood pressure, while in model 2 we adjusted for the variables in Model 1 plus diabetes mellitus, HOMA-IR, cardiovascular morbidity and LDL cholesterol and model 3 included model 2 variables plus alcohol consumption and smoking. In both LTPA-groups, we examined how SAEI changes during the follow up time. Predictive models were built to investigate whether the level of LTPA at visit 1 was associated with levels of LAEI and SAEI at visit 2. Two-way interaction terms showed no differences between men and women in the association between LTPA and SAEI ( $p=0.596$ at visit 1$)$. Thus, we choose to investigate men and women together and to adjust the analysis for gender.

\section{Results}

The average follow-up time was $9.7 \pm 1.4$ years. The average age of the study population at visit 1 was $46.7 \pm 10$ years ( 47.4 for men, 46.2 for women), while being $54.6 \pm 10$ years old at visit 2 (54.8 for men and 54.5 for women). In all, 610 participants reported low and 325 high LTPA at visit 1 . Out of them, 64 persons (6.8\%) were inactive (level 1), 546 (58.4\%) were moderately active (level 2), 294 (31.4\%) were active (level 3) and 31 (3.3\%) were very active (level 4). Based on previous experience [27] and in the low number of subjects in the highest and the lowest level we than dichotomized into 2 groups. Correspondingly, 454 participants reported low and 307 high LTPA at visit 2.

Participants with high LTPA at both visits had lower prevalence in T2DM and hypertension than those with low LTPA. There was a significant difference between the groups of high- and low-LTPA in terms of the mean value for heart rate, and metabolic parameters such as fasting glucose, HOMA-IR, triglycerides (Table 1). No 
Table 1 Characteristics of the participants for visit 1

\begin{tabular}{|c|c|c|c|c|}
\hline & All $n=935$ & Low LTPA $n=610$ & High LTPA $n=325$ & $p$ \\
\hline Age, years (SD) & $46.7(10)$ & $47.5(11)$ & $44.4(10)$ & $0.004^{*}$ \\
\hline Gender, male (\%) & $460(49)$ & $281(61)$ & $179(39)$ & $0.009^{*}$ \\
\hline $\mathrm{BMI}, \mathrm{kg} / \mathrm{m}^{2}(\mathrm{SD})$ & $26.4(4)$ & $26.8(4)$ & $25.5(3)$ & $<0.001^{*}$ \\
\hline $\mathrm{SBP}, \mathrm{mmHg}(\mathrm{SD})$ & $119(14)$ & $119(15)$ & $117(14)$ & 0.055 \\
\hline $\mathrm{DBP}, \mathrm{mmHg}(\mathrm{SD})$ & $69(10)$ & $69(10)$ & $69(9)$ & 0.993 \\
\hline Pulse, bpm (SD) & $63(8)$ & $64(8)$ & $62(8)$ & $<0.001^{*}$ \\
\hline Fasting glucose, mmol/l (SD) & $5.3(0.7)$ & $5.3(0.7)$ & $5.2(0.6)$ & $0.009^{*}$ \\
\hline HOMA-IR median (IQR) & $1.16(0.81-1.78)$ & $1.26(0.88-1.89)$ & $1.09(0.7-1.55)$ & $<0.001^{*}$ \\
\hline Triglyceride, mmol/I median (IQR) & $1.05(0.77-1.46)$ & $1.08(0.79-1.55)$ & $1.01(0.68-1.34)$ & $<0.001^{*}$ \\
\hline LDL cholesterol, mmol/I (SD) & $3.3(0.9)$ & $3.3(0.9)$ & $3.2(0.9)$ & 0.093 \\
\hline CRP, mg/I median (IQR) & $1.3(0.7-2.4)$ & $1.4(0.8-2.6)$ & $1(0.6-1.9)$ & $0.004^{*}$ \\
\hline Diagnosis of hypertension n (\%) & $41(4.4)$ & $30(4.9)$ & $11(3.4)$ & 0.276 \\
\hline Diagnosis of DM n (\%) & $20(2.1)$ & $15(2.5)$ & $5(1.5)$ & 0.355 \\
\hline Cardiovascular morbidity n (\%) & $14(1.5)$ & $11(1.8)$ & $3(0.9)$ & 0.293 \\
\hline Current smoker n (\%) & $151(16)$ & $106(17)$ & $45(14)$ & 0.163 \\
\hline Alcohol n [g/week] median (IQR) & $25.6(9.3-61.5)$ & $24.4(6.3-59.5)$ & $30.1(11.5-67.6)$ & 0.805 \\
\hline SAEI mL/mmHg (SD) & $8.01(3.37)$ & $7.65(3.35)$ & $8.68(3.32)$ & $<0.001^{*}$ \\
\hline LAEI mL/mmHg (SD) & $16.65(4,95)$ & $16.24(4.92)$ & $17.41(4.92)$ & $0.001^{*}$ \\
\hline
\end{tabular}

LTPA: Leisure-Time Physical Activity, p: significance between low and high LTPA, BMI: Body Mass Index, SBP: Systolic Blood Pressure, DBP: Diastolic Blood Pressure, HOMA-IR: Homeostatic Model Assessment for Insulin Resistance, LDL: Low-Density Lipoprotein, DM: Diabetes Mellitus, SAEI: Small Arterial Elasticity Index, LAEI: Large Arterial Elasticity Index, IQR: interquartile range

significant differences between the group with high and low LTPA were observed in LDL cholesterol levels and cardiovascular morbidity (Table 1).

Overall, artery elasticity measured by SAEI and LAEI decreased during the observational time (Visit 1: SAEI $8.01 \pm 3.37 \mathrm{ml} / \mathrm{mmHg}$, LAEI $16.65 \pm 4.95 ; \Delta$ SAEI: 1.41 , CI 1.19-1.61, $p<0.001: \Delta$ LAEI: 1.64, CI 1.29-1.98, $p<0.001$ ). At visit 1 , SAEI was $7.65 \pm 3.35 \mathrm{ml} / \mathrm{mmHg}$ in subjects with low-LTPA and $8.68 \pm 3.32 \mathrm{ml} / \mathrm{mmHg}$ in subjects with high-LTPA $(p<0.001)$, while at visit 2 , SAEI was $6.64 \pm 3.17 \mathrm{ml} / \mathrm{mmHg}$ and $7.71 \pm 3.29 \mathrm{ml} / \mathrm{mmHg}$ $(p<0.001)$, respectively. The same trend was observed in case of LAEI. Specifically, LAEI was $16.24 \pm 4.92$ in individuals with a low level of LTPA and $17.41 \pm 4.92$ in individuals with a high level of LTPA at visit $1(p=0.001)$, and then $15.16 \pm 5.04$ and $16.30 \pm 4.90$ respectively at visit $2(p<0.001)$.

Participants with a high level of LTPA had significantly higher SAEI at visit 1 (Crude model) (SAEI in low-level LTPA: $7.65 \pm 0.14$, SAEI in high-level LTPA: $8.68 \pm 0.19$ $\triangle$ SAEI: 1.03 , CI $0.6-1.15, p<0.001)$ and this difference remained significant after adjustment for possible confounding according to Model 3 (SAEI in low-level LTPA: $7.89 \pm 0.11$, SAEI in high-level LTPA: $8.32 \pm 0.15, \triangle$ SAEI: 0.42 , CI 0.07-0.78; $p=0.020$ ) (Table 2). In model 3 after adjusting for BMI instead of HOMA-IR, similar results were found ( $\Delta$ SAEI: 0.50 , CI: $0.15-0.86 ; p=0.050$ ). No significant association were observed between large vessels elasticity and LTPA after adjustments for Model $1(p=0.690)$.

During the follow up time we observed a decrease in SAEI by $11.9 \%$. The decrease was slightly higher in the group with low LTPA (12.4\%) when compared with the group with high LTPA (11.1\%). However, these differences were not significant. In the longitudinal analyses, we observed that participants with a high LTPA at visit 1 had significantly higher vascular elasticity (SAEI) at visit 2 than subjects with low LTPA ( $\triangle$ SAEI: 1.02 , CI 0.05-1.5, $p=0.022)$ and these associations were strong and significant after adjustments for Model 3 ( $\triangle$ SAEI: 0.44, CI $0.03-0.85, p=0.037$ ) (Fig. 2 and Table 3).

We investigated if the aforementioned associations were different by sex. At both visits, we found that differences between men and women in combination with differences between LTPA levels and SAEI were not significant ( $p=0.596$ at visit $1, p=0.307$ at visit 2 ).

Equivalent analyses regarding LAEI did not show significance $(p=0.286$ at visit $1, p=0.266$ at visit 2$)$.

\section{Discussion \\ Main findings}

In this study, we showed a strong and independent association between leisure-time physical activities and small artery elasticity index independent of possible confounders both at baseline and approximately 10 years later. The association between SAEI and LTPA was strong and 
Table 2 Association between the level of leisure-time physical activity and small artery elasticity index at visit one

\begin{tabular}{|c|c|c|}
\hline \multicolumn{3}{|l|}{$\mathrm{N}=935$} \\
\hline Mean difference & Confidence interval (Cl) & $p$ value \\
\hline \multicolumn{3}{|l|}{ Crude data } \\
\hline $1.03 \mathrm{ml} / \mathrm{mmHg}$ & $0.58-1.48$ & $<0.001$ \\
\hline \multicolumn{3}{|c|}{ Adjusted for age, sex, heart rate and systolic blood pressure } \\
\hline $0.40 \mathrm{ml} / \mathrm{mmHg}$ & $0.05-0.75$ & 0.024 \\
\hline \multicolumn{3}{|c|}{ Adjusted as above and for diabetes mellitus, HOMA-IR, LDL cholesterol and cardiovascular comorbidity } \\
\hline $0.47 \mathrm{ml} / \mathrm{mmHg}$ & $0.12-0.83$ & 0.009 \\
\hline \multicolumn{3}{|c|}{ Adjusted as above + alcohol consumption and smoking } \\
\hline $0.42 \mathrm{ml} / \mathrm{mmHg}$ & $0.07-0.78$ & 0.020 \\
\hline
\end{tabular}

All patients are included who filled in scales about physical activity with baseline and have data for pulse wave analysis with baseline. Patients with known hypertension are excluded

HOMA-IR Homeostatic Model Assessment for Insulin Resistance

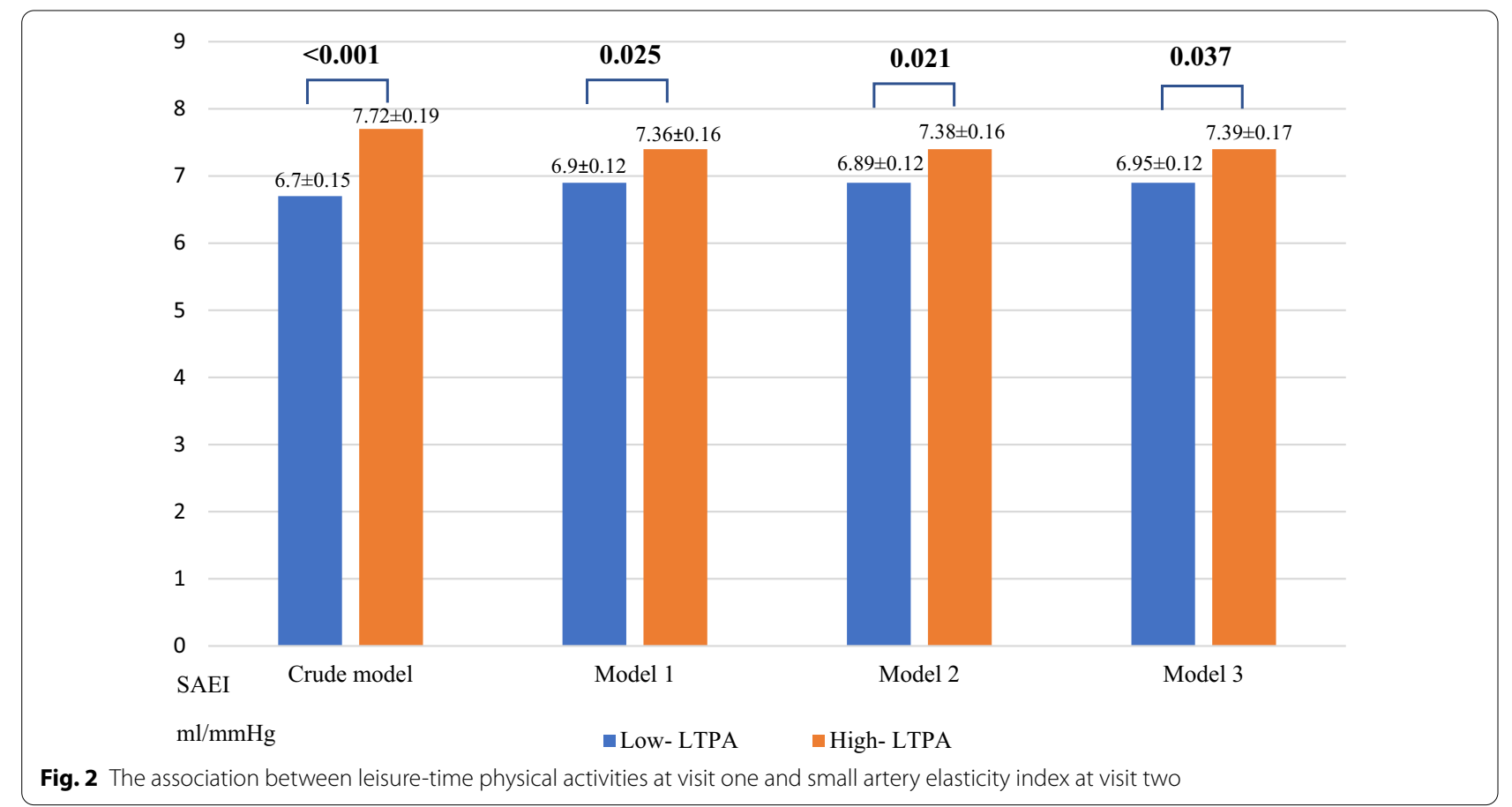

significant even after adjusting in the full model. These results indicate potential salutary effects of exercise on SAEI that may be persistent longitudinally.

Saladini et al. observed the effect of physical activity in 151 subjects with hypertension during a period of 6 years [14]. In line with our findings, they also found that physical activity predicted better SAEI. Moreover, in the Whitehall II study [15], the follow-up time was 5 years and found that moderate-to-vigorous, but not mild, activity was associated with slower progression of aortic stiffness over time. Notably, we were able to include HOMA-IR, diabetes mellitus, cardiovascular morbidity,
LDL cholesterol and alcohol consumption as covariates in the models. Our study confirmed these findings and expanded them even in the non-hypertensive population. To our knowledge this is the first study to investigate the association between reported leisure time physical activities and small artery elasticity index in a middleaged representative cohort of men and women without hypertension.

Physical activity has many benefits both with traditional cardiovascular risk factors and with mortality [28, 29]. In cross-sectional observations, higher artery elasticity is associated with being physically active $[10,12$, 
Table 3 The association between leisure-time physical activities at visit one and small artery elasticity index at visit two, Vara-Skövde cohort

\begin{tabular}{|c|c|c|}
\hline \multicolumn{3}{|l|}{$\mathrm{N}=761$} \\
\hline Mean difference & Confidence interval (CI) & $p$ value \\
\hline \multicolumn{3}{|l|}{ Crude } \\
\hline $1.02 \mathrm{ml} / \mathrm{mmHg}$ & $0.54-1.50$ & $<0.001$ \\
\hline \multicolumn{3}{|c|}{ Adjusted for age, sex, heart rate and systolic blood pressure } \\
\hline $0.46 \mathrm{ml} / \mathrm{mmHg}$ & $0.06-0.86$ & 0.025 \\
\hline \multicolumn{3}{|c|}{ Adjusted as above + diabetes mellitus, HOMA-IR, LDL cholesterol and cardiovascular comorbidity } \\
\hline $0.48 \mathrm{ml} / \mathrm{mmHg}$ & $0.07-0.89$ & 0.021 \\
\hline \multicolumn{3}{|c|}{ Adjusted as above + alcohol consumption and smoking } \\
\hline $0.44 \mathrm{ml} / \mathrm{mmHg}$ & $0.03-0.85$ & 0.037 \\
\hline
\end{tabular}

All patients are included who filled in scales about physical activity with baseline and have data for pulse wave analysis with baseline and follow up. Patients with known hypertension (baseline and follow-up) are excluded

HOMA-IR Homeostatic Model Assessment for Insulin Resistance

13, 30]. In line with these observations, our study found that all indices were significantly better with higher LTPA level at both visits before adjustments.

Small artery elasticity index (SAEI) seems to be strongly associated with changes in the artery wall associated with hypertension [14], diabetes mellitus, and atherosclerosis compared to LAEI [6]. Similarly, it is strongly associated with chronic heart disease, stroke and heart failure [4]. In our study, difference in LTPA levels seems to have a stronger effect on SAEI than LAEI. The stronger association between LTPA levels and SAEI compared with LAEI might depend on that the development of the atherosclerotic disease changes in the endothelial function before structural changes [5] including increased arterial stiffness, particularly in the smaller arteries [6]. In fact, physical activity reduces the plasma endothelin concentration the endothelin mediated vascular tone and thus reduces arterial stiffness [31]. Moreover, physical activity improves endothelial function by up-regulating endothelial nitric oxide synthase (eNOS) protein expression [32]]. Regular physical activity has long-term antiinflammatory effects and improves metabolic control. It is suggested this occurs by skeletal muscle serving as an endocrine organ through a production and secretion of myokines in response to exercise. These mechanisms affect many organs and metabolic pathways, including endothelial cell function [33].

Arterial elasticity indices change over time because of structural modifications in the blood vessels wall like the degeneration of elastin material and an increase in collagenous material and in-ground substance [7]. The factors which can affect the stiffening processes are many, but want to underline ageing, blood pressure, blood glucose, lipids, diseases (hypertension, diabetes mellitus type 1 and 2, coronary artery diseases, heart failure, stroke), medication, lifestyle (smoking, alcohol consumption, physical activity). We have adjusted for these possible confounders in our statistical analysis and found that high level of leisure-time physical activity was still associated with small artery elasticity index suggesting an independent association with these variables.

\section{Strengths and limitations}

All measurements were computed by the same trained staff using a strict protocol providing a strong internal validity in the measurements. The large variety of information and the variables provided by the study permitted the testing of different models. In this study, the inclusion of both men and women made it possible to investigate possible gender differences in the associations and interaction analyses. Even if these differences in SAEI and LAEI between groups with low respective high LTPA are small numerically, these differences are significant at group level and it is consistent with the expected effect of physical activity. A high participation rate at both visits gave higher power to our analyses. A weakness of the study is the use of self-reported physical activity, only providing a subjective estimation of the leisure time physical activities. This scale is however validated and seems to reflect with good approximation the physical activities. Our study was observational without randomization, thereby there is a risk for residual confounding.

\section{Clinical implications}

Cardiovascular prevention is one of the main tasks in primary care. Early introduction of lifestyle change especially physical activity might be important to reduce cardiovascular risk through positive effects on vascular elasticity. Public health strategies introducing leisure-time physical activities early and helping the population to maintain it 
during life should be effective in maintaining good vascular health, reducing the incidence of hypertension and cardiovascular disease.

\section{Conclusions}

We found a positive salutary effect on artery elasticity by physical activity. A higher level of physical activity (LTPA) predicted a higher SAEI in this cohort. Our result suggest that physical activity may inhibit the age-related decline in artery elasticity and arterial compliance.

\section{Acknowledgements}

We want to thank all participants and study nurses for their participation and devotion in this study.

\section{Authors' contributions}

All authors read and approved the final manuscript. Research idea and study design: $\mathrm{BD}, \mathrm{GS}, \mathrm{UL}, \mathrm{MH}$; Data acquisition: $\mathrm{UL}, \mathrm{LR}, \mathrm{BD}, \mathrm{MH}$; Data analysis and interpretation: $\mathrm{BD}, \mathrm{MH}$, GS; Statistical analyses: $\mathrm{BD}, \mathrm{GS}, \mathrm{MA}, \mathrm{UL}, \mathrm{MH}$; Each author contributed important intellectual content during manuscript drafting or revision, accepts personal accountability for the author's own contributions, and agrees to ensure that questions pertaining to the accuracy or integrity of any portion of the work are appropriately investigated and resolved.

\section{Funding}

Open access funding provided by University of Gothenburg. Gabor Szalo: The Skaraborg Institute, Sweden; Funding number: 19/1040; Skaraborg Research and Development Council, Sweden; Funding number:VGFOUSKB-904491 and VGFOUSKB-933331. Bledar Daka: ALF (Funding for medical training and research), Sweden; Funding number: ALFGBG-440441; The Health \& Medical Care Committee of the Region Västra Götaland, Sweden; Funding number: VGFOUREG-862941.

\section{Availability of data and materials}

The datasets used and analyzed during the current study available from the corresponding author on request.

\section{Ethics approval and consent to participate}

All participants were informed on the study procedures and goals and the study obtained written informed consent from all the participants. We confirm that all methods were performed in accordance with relevant guidelines and regulations. This study was approved by the Ethics Committee at the University of Gothenburg, Sweden (DNR: 036-12).

\section{Consent for publication}

No pictures or information that could lead into the identification of the participants were included in this publication. All participants were informed on the scope with the study and the intention to publish the results while keeping personal information confident to the study team and all participants gave written consent to do so.

\section{Competing interests}

We declare that the authors have no competing interests as defined by Journal of BMC Cardiovascular Disorders, or other interests that might be perceived to influence the results and/or discussion reported in this paper.

\section{Author details}

${ }^{1}$ The Skaraborg Institute, Skövde, Sweden. ${ }^{2}$ Primary Health Care, School of Public Health and Community Medicine, Institute of Medicine, Sahlgrenska Academy, University of Gothenburg, Gothenburg, Sweden. ${ }^{3}$ University of California, San Diego, USA. ${ }^{4}$ Department of Clinical Sciences, Lund University, Malmö, Sweden.

Received: 5 November 2020 Accepted: 28 December 2020

Published online: 16 February 2021

\section{References}

1. Kannel WB, Wolf PA, McGee DL, Dawber TR, McNamara P, Castelli WP. Systolic blood pressure, arterial rigidity, and risk of stroke. The Framingham study. JAMA. 1981;245(12):1225-9.

2. Wilson PW, D'Agostino RB, Levy D, Belanger AM, Silbershatz H, Kannel WB. Prediction of coronary heart disease using risk factor categories. Circulation. 1998;97(18):1837-47.

3. Duprez DA, Kaiser DR, Whitwam W, Finkelstein S, Belalcazar A, Patterson R, et al. Determinants of radial artery pulse wave analysis in asymptomatic individuals. Am J Hypertens. 2004;17(8):647-53.

4. Duprez DA, Jacobs DR Jr, Lutsey PL, Bluemke DA, Brumback LC, Polak JF, et al. Association of small artery elasticity with incident cardiovascular disease in older adults: the multi-ethnic study of atherosclerosis. Am J Epidemiol. 2011;174(5):528-36.

5. Duprez DA. Is vascular stiffness a target for therapy? Cardiovasc Drugs Ther. 2010;24(4):305-10.

6. Cohn JN. Arterial compliance to stratify cardiovascular risk: more precision in therapeutic decision making. Am J Hypertens. 2001;14(8 Pt 2):258S-S263.

7. Nilsson PM, Boutouyrie P, Cunha P, Kotsis V, Narkiewicz K, Parati G, et al. Early vascular ageing in translation: from laboratory investigations to clinical applications in cardiovascular prevention. J Hypertens. 2013;31(8):1517-26.

8. O'Rourke MF, Pauca A, Jiang XJ. Pulse wave analysis. Br J Clin Pharmacol. 2001;51(6):507-22.

9. Zimlichman R, Shargorodsky M, Boaz M, Duprez D, Rahn KH, Rizzoni $D$, et al. Determination of arterial compliance using blood pressure waveform analysis with the CR-2000 system: Reliability, repeatability, and establishment of normal values for healthy European population-the seven European sites study (SESS). Am J Hypertens. 2005;18(1):65-71.

10. Zieman SJ, Melenovsky V, Kass DA. Mechanisms, pathophysiology, and therapy of arterial stiffness. Arterioscler Thromb Vasc Biol. 2005;25(5):932-43.

11. Tanaka H, Dinenno FA, Monahan KD, Clevenger CM, DeSouza CA, Seals DR. Aging, habitual exercise, and dynamic arterial compliance. Circulation. 2000;102(11):1270-5.

12. Tanaka H, DeSouza CA, Seals DR. Absence of age-related increase in central arterial stiffness in physically active women. Arterioscler Thromb Vasc Biol. 1998;18(1):127-32.

13. Ashor AW, Lara J, Siervo M, Celis-Morales C, Mathers JC. Effects of exercise modalities on arterial stiffness and wave reflection: a systematic review and meta-analysis of randomized controlled trials. PLoS ONE. 2014;9(10):e110034

14. Saladini F, Benetti E, Mos L, Mazzer A, Casiglia E, Palatini P. Regular physical activity is associated with improved small artery distensibility in young to middle-age stage 1 hypertensives. Vasc Med. 2014;19(6):458-64.

15. Ahmadi-Abhari S, Sabia S, Shipley MJ, Kivimaki M, Singh-Manoux A, Tabak A, et al. Physical activity, sedentary behavior, and long-term changes in aortic stiffness: the Whitehall II Study. J Am Heart Assoc. 2017;6(8):e005974.

16. Lindblad U, Ek J, Eckner J, Larsson CA, Shan G, Rastam L. Prevalence, awareness, treatment, and control of hypertension: rule of thirds in the Skaraborg project. Scand J Prim Health Care. 2012;30(2):88-94.

17. Goransson M, Hanson BS. How much can data on days with heavy drinking decrease the underestimation of true alcohol consumption? I Stud Alcohol. 1994;55(6):695-700.

18. Privsek E, Hellgren M, Rastam L, Lindblad U, Daka B. Epidemiological and clinical implications of blood pressure measured in seated versus supine position. Medicine (Baltimore). 2018;97(31):e11603.

19. Chobanian AV, Bakris GL, Black HR, Cushman WC, Green LA, Izzo JL Jr, et al. Seventh report of the Joint National Committee on Prevention, Detection, Evaluation, and Treatment of High Blood Pressure. Hypertension. 2003;42(6):1206-52.

20. Alberti KG, Zimmet PZ. Definition, diagnosis and classification of diabetes mellitus and its complications. Part 1: diagnosis and classification of diabetes mellitus provisional report of a WHO consultation. Diabet Med. 1998;15(7):539-53.

21. Matthews DR, Hosker JP, Rudenski AS, Naylor BA, Treacher DF, Turner RC. Homeostasis model assessment: insulin resistance and beta-cell function from fasting plasma glucose and insulin concentrations in man. Diabetologia. 1985;28(7):412-9. 
22. Lochen MLRK. The Tromso study: physical fitness, self-reported physical activity, and their relationship to other coronary risk factors. J Epidemiol Community Health. 1992;46:103-7.

23. Wilhelmsen L, Tibblin G, Aurell M, Bjure J, Ekstrom-Jodal B, Grimby G. Physical activity, physical fitness and risk of myocardial infarction. Adv Cardiol. 1976;18:217-30.

24. Morseth B, Hopstock LA. Time trends in physical activity in the Tromso study: an update. PLoS ONE. 2020;15(4):e0231581.

25. Finkelstein SM, Cohn JN. First- and third-order models for determining arterial compliance. J Hypertens Suppl. 1992;10(6):S11-4.

26. Cohn JN, Finkelstein S, McVeigh G, Morgan D, LeMay L, Robinson J, et al. Noninvasive pulse wave analysis for the early detection of vascular disease. Hypertension. 1995;26(3):503-8.

27. Hellgren MI, Larsson CA, Daka B, Petzold M, Jansson PA, Lindblad U. $C$-reactive protein concentrations and level of physical activity in men and women with normal and impaired glucose tolerance. A cross-sectional population-based study in Sweden. J Phys Act Health. 2016;13(6):625-31.

28. Blair SN, Kohl HW 3rd, Barlow CE, Paffenbarger RS Jr, Gibbons LW, Macera CA. Changes in physical fitness and all-cause mortality. A prospective study of healthy and unhealthy men. JAMA. 1995;273(14):1093-8.
29. Nocon M, Hiemann T, Muller-Riemenschneider F, Thalau F, Roll S, Willich SN. Association of physical activity with all-cause and cardiovascular mortality: a systematic review and meta-analysis. Eur J Cardiovasc Prev Rehabil. 2008;15(3):239-46.

30. O'Donovan C, Lithander FE, Raftery T, Gormley J, Mahmud A, Hussey J. Inverse relationship between physical activity and arterial stiffness in adults with hypertension. J Phys Act Health. 2014;11(2):272-7.

31. Maeda S, Sugawara J, Yoshizawa M, Otsuki T, Shimojo N, Jesmin S, et al. Involvement of endothelin-1 in habitual exercise-induced increase in arterial compliance. Acta Physiol (Oxf). 2009;196(2):223-9.

32. Green DJ, Maiorana A, O'Driscoll G, Taylor R. Effect of exercise training on endothelium-derived nitric oxide function in humans. J Physiol. 2004;561(Pt 1):1-25.

33. Severinsen MCK, Pedersen BK. Muscle-organ crosstalk: the emerging roles of Myokines. Endocr Rev. 2020;41(4):594-609.

\section{Publisher's Note}

Springer Nature remains neutral with regard to jurisdictional claims in published maps and institutional affiliations.
Ready to submit your research? Choose BMC and benefit from:

- fast, convenient online submission

- thorough peer review by experienced researchers in your field

- rapid publication on acceptance

- support for research data, including large and complex data types

- gold Open Access which fosters wider collaboration and increased citations

- maximum visibility for your research: over 100M website views per year

At BMC, research is always in progress.

Learn more biomedcentral.com/submissions 